\title{
Pathological Alterations in Dogs Resulting from Parasitism by Spirocerca Lupi
}

\author{
Fabiana Elias', Rafaela Magalhães Barros², Helvecio Leal Santos-Junior², Rômulo Santos Adjuto Eloi³, \\ Vinicius Silva ${ }^{4}$, Fagner Freitas' \& Carlos Eduardo Fonseca-Alves ${ }^{5}$
}

\begin{abstract}
Background: Spirocercosis is considered to be an emerging pathological condition within veterinary medicine. The disease has worldwide distribution, although the greatest prevalence has been described in tropical and subtropical regions. The infection rate is affected by the interrelationships between the density of the dog population and intermediate hosts. The aim of this study was report the morphological and histopathological characteristics of natural infection by Spirocerca lupi in dogs from Distrito Federal, Brazil.

Cases: Records were obtained from 10 dogs diagnosed with spirocercosis at the Pathology Sector, School of Veterinary Medicine, União Pioneira de Integração Social (UPIS), in Brasília, Federal District. For six animals, the diagnosis was confirmed through necropsy. Two dogs presented aortic rupture, thus resulting in hemothorax and consequent hypovolemic shock. Another animal presented gastric nodules, erratic migration to the lungs and renal and splenic infarction due to aortic thromboembolism. One dog presented neoplastic transformation of the esophageal granuloma to osteosarcoma. The age of the dogs ranged from one to eight years and the weight ranged from 15 to $40 \mathrm{~kg}$. The clinical histories were similar: regurgitation (10/10), emesis (5/10), dysphagia (7/10), weight loss (10/10), weakness (3/10) and dyspnea (4/10). Esophageal nodules measured 1 to $5 \mathrm{~cm}$ in diameter were observed in all the dogs and contained a large number of Spirocerca lupi specimens. Histologically, parasites were detected in adventitial and medial areas, surrounded by zones of necrosis and mixed inflammatory infiltrate. The stroma of the intimal tissue presented considerable fibrocellular proliferation (atherosclerosis), with areas of mineralization and ossification in the intimal and medial layers. An interesting finding of this study was atypical migration to the stomach and osteosarcoma case associated with parasitism by Spirocerca lupi. Discussion: Infection by Spirocerca lupi is very important in Brazil, however, there are few studies showing epidemiological, morphological and histopathological aspects of the parasite infection. In the Distrito Federal a large number of animals has died due to rupture of nodules located in the thoracic aorta, however, necropsy was not performed and these animals do not present confirmed diagnosis. Our results showed a major group of animals of large breed (6/10) follow by medium (3/10) and small (1/10) breeds. The incidence of spirocercosis is greater in medium to large-sized dogs because these animals remain outside of the domestic area for longer times than do small-sized animals. In this study, there was greater prevalence in mongrels. Nonetheless, breeds such as Cocker Spaniel, Borzoi, German Shepherd and Great Dane have also been affected by this condition. In general, the majority of free-living dogs are mongrels and the parasitosis rate is high among dogs of this type in enzootic areas. The great prevalence of adult dogs is related to the characteristics of the parasite's life cycle, along with difficulty in making the diagnosis in animals under six months of age. We did not found young dogs showing infection by Spirocerca lupi. The age range between four and twelve years is most affected. Although the number of dogs diagnosed with spirocercosis has been increasing continuously in the Distrito Federal, it is commonly only identified through necroscopic examinations. Therefore, measures need to be taken towards making early diagnosis and appropriate treatment, in order to reduce the death rate. Due to the scarcity of data in the Brazilian literature about this condition, the present study and future studies and data-gathering are of prime importance.
\end{abstract}

Keywords: aneurysms, dogs, necropsy, Spirocerca lupi.

${ }^{1}$ Laboratório de Patologia Veterinária, Universidade Federal da Fronteira Sul (UFFS), Realeza, PR, Brazil. ${ }^{2}$ Setor de Patologia, Faculdade de Medicina Veterinária da União Pioneira de Integração Social (UPIS), Brasília, DF, Brazil. ${ }^{3}$ Laboratório HistoPato, Brasília. ${ }^{4}$ Escola de Medicina Veterinária e Zootecnia, Universidade Federal do Tocantins (UFT), Araguaína, TO, Brazil. ${ }^{5}$ Department of Veterinary Clinic, School of Veterinary Medicine and Animal Science, Universidade Estadual Paulista (UNESP), Botucatu, SP, Brazil. CORRESPONDENCE: C.E. Fonseca-Alves [carloseduardofa@ hotmail. com - Tel.: +55 (14) 38802076]. School of Veterinary Medicine and Animal Science - UNESP. CEP 18609-020 Botucatu, SP, Brazil. 


\section{INTRODUCTION}

Spirocercosis is considered to be an emerging pathological condition within veterinary medicine [2]. Its etiological agent is the nematode Spirocerca lupi. This parasite mainly affects dogs, but it may also affect other animals, particularly wild canids $[5,6]$.

The disease has worldwide distribution, although the greatest prevalence has been described in tropical and subtropical regions [2,4-6,11]. Recent reports on canine spirocercosis have come from Israel, Greece, Iraq, Iran, India, Malaysia, South Africa, Brazil, the Caribbean, Mexico and the United States [1]. The infection rate is affected by the interrelationships between the density of the dog population and intermediate hosts [4].

Lesions due to Spirocerca lupi mostly originate from migration and persistent presence of the larvae and adults in tissues $[2,4,11]$. If the granulomas are cut open, numerous live red nematodes can be seen [9]. Controlling this parasite is very difficult because of the ubiquitous nature of the intermediate and paratenic hosts [5]. Given the well-known evolution of the prevalence of canine spirocercosis and the multi-systemic alterations resulting from this, along with the scarcity of Brazilian epidemiological data, the aim of this study was report the morphological and histopathological characteristics of natural infection by Spirocerca lupi in dogs from Distrito Federal, Brazil.

\section{CASES}

Records were made regarding dogs diagnosed with spirocercosis at the Pathology Sector of the School of Veterinary Medicine, União Pioneira de Integração Social (UPIS), in Brasília, Federal District, between July 2005 and August 2007. A total of 10 cases in different breeds were thus recorded, with ages between one and eight years and weights between 15 and 40 $\mathrm{kg}$. All the medical files of these animals were examined with the aim of describing the main clinical signs presented and the possible therapeutic interventions.

For six animals, the diagnosis was confirmed through necropsy. Two dogs presented aortic rupture, thus resulting in hemothorax and consequent hypovolemic shock. Another animal presented gastric nodules, erratic migration to the lungs and renal and splenic infarction due to aortic thromboembolism. One dog presented neoplastic transformation of the esophageal granuloma to osteosarcoma.

During the necropsy, the organs were exposed for subsequent morphological evaluation. If the presence of nodules was detected, tissue samples were collected from the thoracic aorta and esophagus: these were fixed in $10 \%$ formaldehyde and placed in individual flasks, which were labeled specifying the scientific name of the species, the location and the date of the necropsy. Subsequently, the material was sent to the histopathology laboratory of UPIS.

The organs were kept in the fixing solution ( $10 \%$ formaldehyde) for a minimum period of $24 \mathrm{~h}$. After this, the samples were subjected to tissuel processing, consisting of dehydration in a series of alcohols of increasing purity, clearing in Xylene and embedment in paraffin, following routine laboratory procedures [2]. Semi-serial sections of $\mu \mathrm{m}$ in thickness were obtained and then stained with hematoxylin-eosin. The microscopic images were recorded by means of a digital eyepiece, coupled to a Bioval binocular microscope, and were stored in a computer.

The age of the dogs that were subjected to necropsy ranged from one to eight years and the weight was a ranged from 15 to $40 \mathrm{~kg}$. The clinical histories of these animals were similar and the animals showed: regurgitation (10/10), emesis (5/10), dysphagia (7/10), weight loss (10/10), weakness (3/10) and dyspnea (4/10). Esophageal nodules measured 1 to 5 centimeters in diameter were observed in all the dogs (10/10) (Figure 1A). When these nodules were cut open, we found a large number of Spirocerca lupi specimens. Histologically, parasites were detected in adventitial and medial areas, surrounded by zones of necrosis and mixed inflammatory infiltrate (Figure 1B). The stroma of the intimal tissue presented considerable fibrocellular proliferation (atherosclerosis), with areas of mineralization and ossification in the intimal and medial layers.

We found transmural orifices showing $2 \mathrm{~cm}$ in diameter, with irregular borders close to the base of the heart in the thoracic aorta of two animals. The cause of these animals' deaths were defined as hypovolemic shock due to aortic rupture. An interesting finding of this study was atypical migration of parasites to different organs. An eight-year-old mongrel showed a gastric and pulmonary nodule wit 1 and $2 \mathrm{~cm}$ in diameter re- 
spectively (Figure 2A). When we the cut of the nodules was possible to identify a large number of parasites.

An 8-year-old mongrel dog showed abdominal hemorrhage and it observed at necropsy the spleen with a dark red color and was enlarged, measuring $50 \mathrm{~cm}$ in length, with several rupture points in the capsule and a focally extensive area of infarction. The kidneys present tumefaction (Figure 2C) and when cut open showed a prominent triangular area of infarction of dark red color, which extended from the cortex to the medulla. The renal

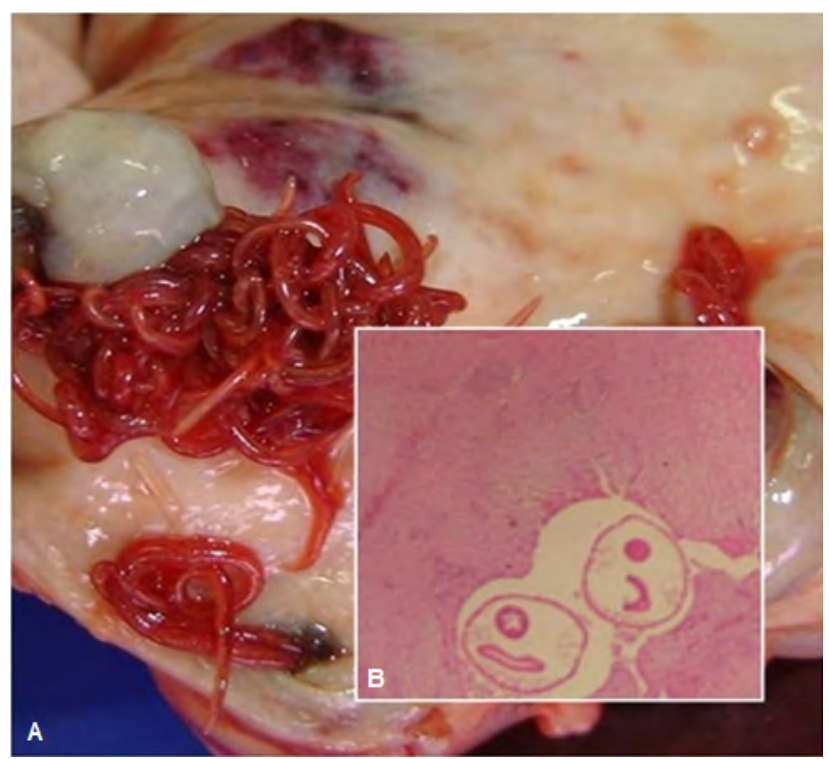

Figure 1. A- Longitudinal section through the esophagus of a dog with spirocercosis. Note the formation of nodules that, when cut open, revealed large numbers of parasites compatible with Spirocerca lupi. B- Photomicrograph of the esophagus. The parasitic forms are seen to be surrounded by fibroblasts and inflammatory infiltrate [H.E. 100x].

\section{DISCUSSION}

Infection by Spirocerca lupi is very important in our country, however, there are few studies showing epidemiological, morphological and histopathological aspects of the parasite infection in Brazil. In our experience, in the Distrito Federal a large number of animals has died due to rupture of nodules located in the thoracic aorta, however, necropsy was not performed and these animals do not present confirmed diagnosis. Our results showed a major group of animals of large breed (6/10) follow by medium (3/10) and small (1/10) and splenic infarctions were associated with aortic thromboembolism (Figure 2D).

In the histopathological evaluation of the esophageal granuloma, only one animal showed a neoplastic transformation to osteosarcoma (Figure 2B). Histologically, there was marked mononuclear infiltrate surrounded by abundant bundles of compact connective tissue, which in some areas were bordering the parasite, as well as formation of bone matrix with neoplastic differentiation, coming from the esophageal granuloma.

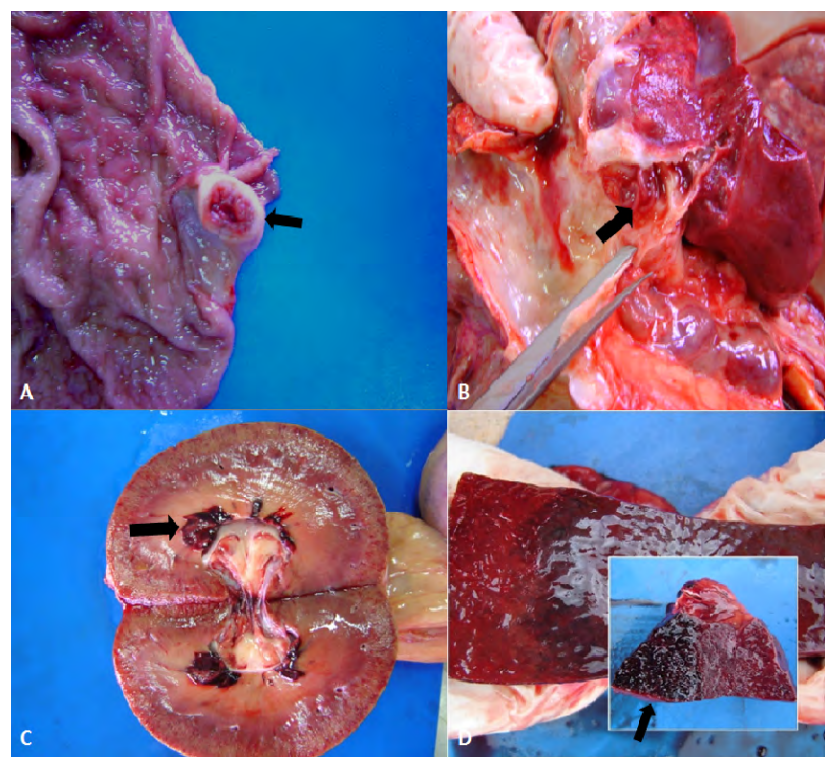

Figure 2. A- Stomach nodule showing $2 \mathrm{~cm}$ of diameter (arrow) in an eight-year-old mixed breed dog containing parasitic forms of Spirocerca lupi. B- Formation of mineralized matrix, characterizing neoplastic transformation of the esophageal granuloma into osteosarcoma. C- Kidney of a dog with spirocercosis. The organ presents tumefaction and has an area of infarction in the medulla (arrow), resulting from aortic thromboembolism. D- Spleen of a dog with spirocercosis. The organ presents increased volume, measuring $50 \mathrm{~cm}$ in length, with several points of capsule rupture and a focally extensive area of infarction (inserti - arrow), resulting from aortic thromboembolism.

breeds. The incidence of spirocercosis is greater in medium to large-sized dogs because these animals remain outside of the domestic area for longer times than do small-sized animals, thus facilitating infection of larger dogs by the agent [10].

In this study, there was greater prevalence in mongrels, thus corroborating what was described in the literature [1]. Nonetheless, breeds such as Cocker Spaniel, Borzoi, German Shepherd and Great Dane have also been affected by this condition. In general, the majority of free-living dogs are mongrels and, the parasitosis rate is high among dogs of this type in en- 
zootic areas $[1,11]$. The greater prevalence adult dogs is related to the characteristics of the parasite's life cycle, along with difficulty in making the diagnosis in animals under six months of age [11]. We did not found young dogs showing infection by Spirocerca lupi. The age range between four and twelve years is most affected $[9,10]$.

The clinical signs presented by the animals were related to esophageal nodules causing obstruction of the lumen or rupture of nodules in the thoracic aorta region. Regurgitation and vomiting were the most common clinical signs in our study. These clinical signs were compatible with those described by others authors [4-6,8,10-12]. Histopathological diagnosis confirmed infection with the parasite in all the cases. Histologically, mature esophageal nodules were characterized by abundant bundles of compact connective tissue containing eosinophilic and lymphocytic inflammatory infiltrate bordering the parasites, similar to what was described by Van Der Merwe et al. [12].

One constant finding was based on the aorta, which was irregular, containing firm multifocal thinning and severe multifocal dilatation compatible with aneurysms. In some segments, firm reddish points were seen. Aortic scarring, aneurysms and esophageal granulomas are lesions that are more frequently seen and are considered to be pathognomonic for spirocercosis [7-10]. Aneurysms are due to debility and loss of elasticity, associated with thickening of the artery, due to the inflammatory process that is stimulated by migration of the parasite through the intimal layer of the vessel. Recently, the capacity to produce nitric acid and/or other oxygen-reactive metabolites involved in controlling vascular tonus was correlated with infection by Spirocerca lupi. These substances might aid in the development of aortic aneurysms [7,11].

Two animals were necropsied due to sudden death. These animals have a history of sporadic regurgitation and the owners have not sought veterinary care to seek the cause of this clinical sign. These animals came to death due to hypovolemic shock due to aortic rupture. Cardiovascular shock or collapse gives rise to systemic hypoperfusion because of the reduction in cardiac output or circulating blood volume. The final results are hypotension followed by reduction of tissue perfusion and cell hypoxia [11]. The mucosa of these animals acquires a pearly appearance. Lavy et al. [5] stated that animals with spirocercosis could present sudden death as a result of rupture of aortic aneurysms. Because of hemothorax, approximately three liters of partially coagulated blood could be seen in the cavity.

It was found an aberrant migration of the parasite to lung and stomach. In consonance with this description, Ranen et al. [10] stated that parasites could be found in granulomas or nodules in this region. Aberrant migrations of Spirocerca lupi are common, and larvae can be found in different organs, particularly those of the thoracic cavity [2]. Lesions can also occur less frequently due to aberrant migration of larvae [6]. Alterations to the blood flow due to aortic aneurysms predispose towards formation of thrombi. These thrombi may become dislodged and then occlude the blood vessels that branch out from the aorta [2].

One patient had osteosarcoma associated with infection by the parasite. The occurrence of tumors due to infection by Spirocerca lupi is as low as squamous cell and leiomyosarcoma the most common diagnosis. On rare occasions, fibrosarcoma and osteosarcoma of the esophagus may develop after malignant transformation inside the wall of the esophageal granulomas, associated with infection with Spirocerca lupi [9]. Esophageal sarcomas presumably occur in progressive chronic cases. One supposition is the elevated age of the dogs that were seen to have esophageal sarcomas. This can be explained by the greater duration of time required for granulomas to become neoplastic [10-12]. The age of the dog in this report was unknown, but from the condition of the dental arch, it was an elderly animal. It was a female and, this sex is at greater risk of developing spirocercosis associated with esophageal sarcoma [10]. Nonetheless, this author suggested that this finding should be investigated in a greater number of cases. The animal of the present report had pale mucosa and a complete blood count showed anemia with normal cells and normal coloration. Absolute neutrophilia is the commonest hematological abnormality. This alteration may be caused by an inflammatory reaction associated with the tumor [10].

The pathogenesis of spirocercosis in inducing neoplasms has not been well elucidated [4-6]. Esophageal sarcomas in dogs are almost exclusively found in areas that are endemic for parasitism due to Spirocerca lupi, which suggests that they are induced by the larvae [10]. There are hypotheses that parasites may interfere with cellular processes of relevance to carcinogenesis, through acting in relation to intercel- 
lular communication or even through secretion of molecules with carcinogenic potential [11].

Although the number of dogs diagnosed with spirocercosis has been increasing continuously in the Distrito Federal, it is commonly only identified through necroscopic examinations. Therefore, measures need to be taken towards making early diagnosis and ap- propriate treatment, in order to reduce the death rate. Due to the scarcity of data about this condition in the Brazilian literature, future studies with data-gathering are of prime importance.

Declaration of interest. The authors report no conflicts of interest. The authors alone are responsible for the content and writing of the paper

\section{REFERENCES}

1 Chikweto A., Bhaiyat M.I., Tiwari K.P., Allie C. \& Sharma R.N. 2012. Spirocercosis in owned and stray dogs in Grenada. Veterinary Parasitology. 190(3-4): 613-616.

2 Gal A., Kleinbart S., Aizenberg Z. \& Baneth G. 2005. Aortic thromboembolism associated with Spirocerca lupi infection. Veterinary Parasitology. 130(3-4): 331-335.

3 Herrera L.A. \& Ostrosky-Wegman P. 2001. Do helminths play a role in carcinogenesis? Trends Parasitology. 17(4): $172-175$.

4 Klainbart S., Mazaki-Tovi M., Auerbach N., Aizenberg I., Bruchim Y. \& Dank G. 2007. Spirocercosis - associated pyothorax in dogs. Veterinary Journal. 173(1): 209-214.

5 Lavy E., Aroch I., Bark H., Markovics A., Aizenberg I. \& Mazaki-Tovi M. 2002. Evaluation of doramectin for the treatment of experimental canine spirocercosis. Veterinary Parasitology. 109(1-2): 65-73.

6 Mazaki-Tovi M., Baneth G., Aroch I., Harrus S., Kass P. \& Ben-Ari T. Canine spirocercosis: clinical, diagnostic, pathologic, and epidemiologic characteristics. Veterinary Parasitology. 107(3): 235-250.

7 Meléndez R. \& Suárez-Pellín C. 2001. Spirocerca lupi and dogs: the role of nematodes in carcinogenesis. Trends Parasitology. 17(11): 516.

8 Mylonakis M.E., Ceronb J.J., Leontidesc L., Rallisa T. S. \& Koutinas A.F. 2012. Serum acute phase proteins in dogs with symptomatic esophageal spirocercosis. Veterinary Parasitology. 190(1-2): 191-195.

9 Pazzi P., Tompkins S. \& Kirberger R.M. 2013. Canine spirocercosis-associated extraskeletal osteosarcoma with central nervous system metastasis. Journal of South African Veterinary Association. 84(1): 1-4.

10 Ranen E., Lavy E., Aizenberg I., Perl A. \& Harrus S. 2004. Spirocercosis - associated esophageal sarcomas in dogs. A retrospective study of 17 cases (1997-2003). Veterinary Parasitology. 119(2-3): 209-221.

11 Santos A.S.O., Silveira L.S., Lemos L.S., Moreira L., Silva A.C. \& Carvalho E.C.Q. 2004. Aneurismas aórticos pela espirocercose canina em Brasília (Relato de caso). Revista Brasileira de Saúde e Produção Animal. 5(1): 25-30.

12 Van Der Merwe L.L., Kirberger R.M., Clift S., Williams M., Keller N. \& Naidoo V. 2008. Spirocerca lupi infection in the dog: a review. Veterinary Journal. 176(3): 294-309. 